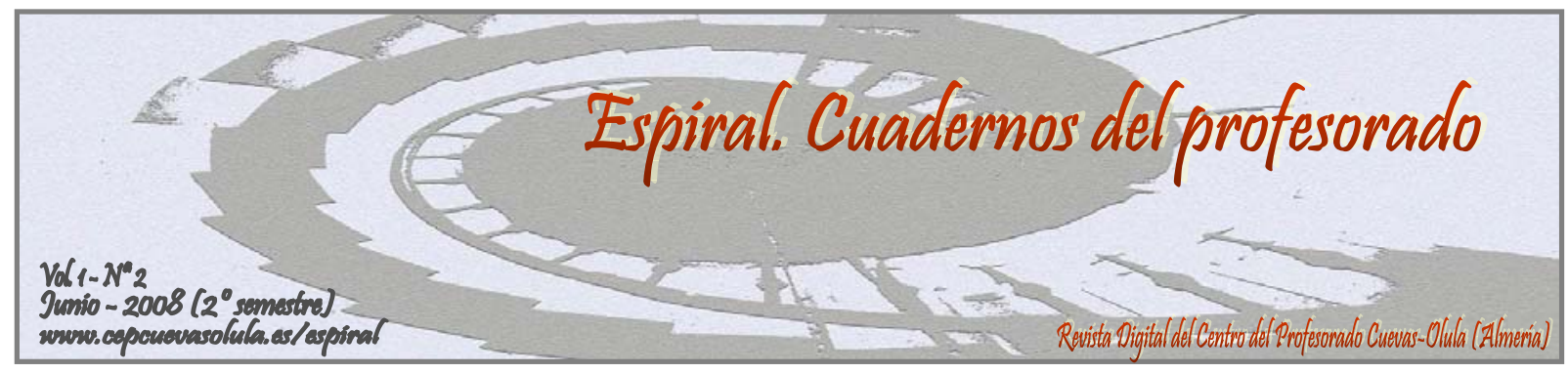

\title{
CONFECCIÓN DEL PLAN DE TRABAJO DEL ESPECIALISTA DE PEDAGOGÍA TERAPÉUTICA: UN EJEMPLO PRÁCTICO.
}

\author{
José Antonio Torres González, María Jesús Colmenero Ruiz \\ y Antonio Hernández Fernández
}

Universidad de Jaén

Enviar correspondencia a: ahernand@ujaen.es

\begin{abstract}
RESUMEN: La realidad educativa de la que partimos exige al maestro de pedagogía terapéutica la elaboración de un Plan de Trabajo, entendido éste como modelo sistemático de actuación pública o privada, que se elabora anticipadamente para dirigirla y encauzarla. La realización del Plan de Trabajo surge de la necesidad de planificar las actuaciones que se vayan a llevar a cabo en el centro en relación a la Atención a la Diversidad desarrollándose en cada uno de los puntos. Desarrollaremos en este artículo la estructura organizativa para atender a la diversidad. Especificaremos las actuaciones de planificación, coordinación, asesoramiento, previsión y elaboración de material que será necesario para crear una escuela integradora de necesidades y no limitadora de oportunidades. En estas actuaciones se concretarán los lazos de unión entre toda la Comunidad Educativa, estrechándose la colaboración entre familia, escuela, servicios externos y la sociedad en general. Nuestro Plan de Trabajo se concretará para Lucía, una alumna con una serie de cualidades que le hacen ser "especial". Todo lo nombrado se concretará en quince Unidades Didácticas de marcado carácter curricular que serán adaptadas a las características que presenta Lucía.
\end{abstract}

Palabras clave: plan de trabajo, programación, diversidad, integración, educación.

ABSTRACT: The educational reality of which we leave demands the teacher of therapeutic pedagogy the elaboration of a Plan of Work, expert this like systematic model of public or private performance that it is elaborated in advance to direct it and to channel it. The realization of the Plan of Work arises of the necessity of planning the performances that will carry out in the center in relation to the Attention to the Diversity being developed in each one of the points. We will develop in this article the organizational structure to assist to the diversity. We will specify the performances of planning, coordination, advice, forecast and material elaboration that it will be necessary to create an integrative school of necessities and not constrainer of opportunities. In these performances the knots of union will be summed up among the whole Educational Community, being narrowed the collaboration among family, school, external services and the society in general. Our Plan of Work will be summed up for Lucía, a coed with a series of qualities that you/they make him be "special". All that named will be summed up in fifteen Didactic Units of marked curricular character that will be adapted to the characteristics that Lucía presents.

Key words: work plan, programming, diversity, integration, education. 


\section{1.- INTRODUCCIÓN.}

La realización de un Plan de Trabajo surge de la necesidad de planificar las actuaciones que se vayan a llevar a cabo en el centro en relación a la Atención a la Diversidad desarrollándose en cada uno de los puntos.

La estructura organizativa para Atender a la Diversidad es un punto importante que vamos a desarrollar: ¿Qué profesionales y Equipos se encargarán de atender específicamente las Necesidades Educativas Especiales de los alumnos? ¿Cuáles son sus funciones? ¿Cómo se organizan?

Una vez que conocemos quienes son los profesionales especializados que trabajan con los alumnos con Necesidades Educativas Especiales hemos de especificar las actuaciones de planificación, coordinación, asesoramiento, previsión y elaboración de material que será necesario para crear una escuela integradora de necesidades y no segregadora de oportunidades. En estas actuaciones se concretarán los lazos de unión entre toda la Comunidad Educativa, estrechándose la colaboración entre familia, escuela, servicios externos y la sociedad en general.

Nuestro Plan de Trabajo se ejemplificará en "Lucía", una alumna con una serie de cualidades que le hacen ser especial, entre ellas hemos de destacar que es una niña cariñosa, sociable, siempre busca el afecto de los demás, es perseverante en sus tareas y suele ser muy presumida. Siempre tiene ganas de aprender pero se aburre muy pronto, por lo que siempre hay que estar cambiándole las tareas. Estos y otros motivos son los que han hecho que escoja a esta niña, por ser una persona que despierta la curiosidad e interés.

\section{2.- JUSTIFICACIÓN.}

¿Por qué tiene un centro ordinario que realizar un Plan de Trabajo? ¿Qué relación tiene el Plan de Trabajo con los diferentes niveles de concreción del centro? ¿Cómo se planifica la Respuesta Educativa desde el mismo? y ¿Qué funciones desempeña el maestro de trabajo a la integración al respecto?

El Plan de Trabajo se considera como una de las medidas de Atención a la Diversidad, esto quiere decir que podemos justificar la realización del mismo como organizador de las actuaciones necesarias para el análisis de las necesidades que se presentan en el centro y más concretamente en los alumnos con Necesidades Educativas Especiales.

La planificación de la Respuesta Educativa para los alumnos con Necesidades Educativas Especiales parte de la necesidad de la realización de un análisis pormenorizado de las características tanto personales como del entorno en el que se desenvuelven nuestros alumnos y del contexto escolar. Esta respuesta específica se concreta en las actuaciones que realizaremos con los alumnos con Necesidades Educativas Especiales tanto dentro como fuera del aula ordinaria, en grupo o a nivel individual, mediante el refuerzo educativo en una o varias áreas instrumentales, siempre teniendo en cuenta las orientaciones del Equipo de Orientación Educativa así como de otras instituciones especializadas en la materia.

\section{3.- MARCO TEÓRICO CONCEPTUAL.}

Para comprender a que y a quienes nos referimos cuando hablamos del plan de apoyo, hemos de saber qué entendemos por diversidad. Cuando hablamos de diversidad, hacemos referencia a la variedad de intereses, ritmos, capacidades y motivaciones de los alumnos, así como, a las necesidades educativas de distinto orden que puedan presentar por condiciones personales de discapacidad o por causas sociales desfavorecidas o una historia de aprendizajes desajustada. Este nuevo concepto de atención a la diversidad. Para llevar a cabo esta idea de atención a las necesidades e intereses de los alumnos de forma adecuada, planteamos la idea de una escuela de carácter comprensivo e integrador, en la cual se integren necesidades y no niños, a través de la atención y apoyo personalizados, con diversificaciones curriculares, incluso con determinados agrupamientos coyunturales... siempre con el objetivo de lograr la integración de dichos estudiantes, siempre transmitiendo expectativas positivas e impulsando y apoyando los avances.

Además para saber a quienes nos referimos cuando hablamos de alumnos con necesidades educativas especiales, debemos plantear que entendemos por necesidades educativas especiales. Así con la L.O.E., se establece que el término N.E.E. se encuentra estrechamente ligado al de Dificultades de Aprendizaje, es decir, que existe cuando hay una dificultad para aprender significativamente superior que la mayoría de los niños de su misma edad y /o sufre de alguna alteración que le impide o dificulta el 
uso de los recursos educativos que generalmente tienen a su disposición los compañeros de su misma edad. Las necesidades educativas se extienden a lo largo de un continuo, que va desde las necesidades ordinarias, planteadas para la mayoría de los alumnos/ as, que no precisan de recursos especiales, hasta las necesidades más específicas o necesidades educativas que poseen algunos alumnos, y que precisan de determinadas ayudas o servicios extras.

No sería justo hacer una fundamentación teórica sin tener en cuenta a autores de la talla de Pestalozzy (1801) y Fröebel (1826) que fueron los introductores de unos nuevos principios en los que basar la educación en general y la E.E. en particular. Estos principios son: globalización, auto-educación, socialización y actividad La respuesta a nuestros alumnos vendría dada por la concepción de la reforma del Sistema Educativo que apoya al constructivismo, que se entiende como una postura que destaca el papel activo del estudiante en la estructuración del conocimiento y dar sentido a la información. Destacando autores como Vigosky (1985), Norman (1987), Ausubel (1975), Piaget (1981), etc...

Igualmente nos fundamentaremos en el paradigma ecológico porque el niño necesita estar inmerso en los conflictos del grupo clase como sistema social, para las actividades cognitivas y afectivas que se desarrollan en el intercambio académico. $Y$ en el paradigma cognitivo, por la adquisición significativa de los conocimientos, la formación de estructuras internas y el desarrollo de estrategias operativas de pensamiento y ejecución (Doyle, 1977).

\section{4.- ANÁLISIS DEL CONTEXTO.}

\section{General.}

Los edificios del centro rodean los dos patios de que disponemos: el patio de Educación Infantil que se sitúa a la izquierda de su edificio. Es un patio de tierra con columpios, toboganes... en una pequeña porción de ese patio se ha hecho un pequeño circuito donde se han puesto las señales de tráfico más comunes. Circuito que podríamos utilizar el aula de trabajo y el aula específica. El otro, es un patio muy amplio en el que encontramos dos pistas, una es de fútbol y baloncesto con sus respectivas canastas y porterías. La otra pista se suele utilizar para jugar a voleibol puesto que no dispone nada más que de las líneas pintadas en el suelo. Las dos pistas dejan un espacio amplio para que los que quieran jugar a otras cosas puedan hacerlo aun estando ocupadas las dos pistas.

Todas las aulas dan al exterior con grandes ventanas que proporcionan gran iluminación, estas son de doble acristalamiento para aislar del ruido.

El material a destacar de las aulas de nuestro centro, además del que todos conocemos, son: la disposición de espejos en las aulas de Educación Infantil y Primer ciclo de Educación Primaria encaminados a trabajar la identidad personal y el conocimiento del propio cuerpo. Objetivos que a en algunas ocasiones habremos de trabajar con nuestros alumnos con N.E.E. Los recursos materiales de que dispone cada nivel están en función de los objetivos didácticos que se vayan a trabajar (Ejemplo en Educación Infantil y primer ciclo de Educación Primaria cuentan con puzzles, muñecos articulados, encajables, lotos fotográficos de expresiones...).

Los profesores que componen nuestro centro son veintisiete, de ellos dieciocho generalistas, dos especialistas en educación física, dos maestros de música, dos de inglés, dos maestros de pedagogía terapéutica (un maestro de pedagogía terapéutica de trabajo a la integración y maestro de pedagogía terapéutica de aula específica) y un logopeda. Hay interinos, maestros acaban de aprobar las oposiciones y no tienen destino y los demás tienen ya su destino en este centro. Tenemos que destacar que el profesorado de este centro aboga por la integración de los alumnos con NEE, con lo que su colaboración siempre es activa y continua entre ellos y los maestros de Trabajo a la Integración y de audición y lenguaje.

El centro tiene escolarizados 400 alumnos. El nivel de absentismo en nuestro centro es muy reducido, existe un nivel de conciencia elevado entre las familias de la necesidad de acudir a la escuela. Hemos de destacar que hay alumnos que tienen poca motivación para el aprendizaje.

Nuestro principal interés en este Plan de Trabajo será resaltar la presencia de alumnos con NEE, alumnos cuyo proceso de evaluación está en marcha para detectar las NEE y por último, los alumnos que no presentan NEE pero tienen dificultades de aprendizaje, con el objeto de darle a cada cual la Respuesta Educativa adecuada a sus características.

Las familias de los alumnos del centro se caracterizan por estar muy sensibilizadas hacia la necesidad y la importancia de la educación como uno de los pilares básicos para la formación 
integral de la persona. El nivel socioeconómicocultural de las familias es medio-bajo. A nivel general, podemos decir que su nivel de participación es bastante bueno. Su concienciación en cuanto a la necesidad de mantener una relación estrecha entre la escuela y la familia es muy elevada, en parte por el trabajo realizado por el Equipo Educativo. En nuestro centro también encontramos familias provenientes de otros países, con una cultura diferente $\mathrm{y}$ a la que hemos de respetar $\mathrm{e}$ introducir entre los escolares.

\section{Contexto específico: unidad de pedagogía terapéutica}

El aula de Apoyo a la Integración está situada según entramos al centro al fondo a la izquierda, en un edificio de dos plantas donde se encuentra el primer ciclo de primaria y teniendo justo enfrente a los alumnos de Educación Infantil. Está ubicada en la primera planta del inmueble al lado del aula específica de Educación Especial, de logopedia y el aula de recursos.

El aula es pequeña, sus ventanas dan al exterior, por los que está provista de bastante luminosidad, con doble acristalamiento para aislar lo más posible el ruido del exterior. El mobiliario está dispuesto de manera que haya suficiente espacio para movernos con total libertad, de baja altura para que los materiales que haya en ellos puedan cogerlos todos los alumnos sin ningún problema. Tenemos siete mesas y de ellas dos son adaptadas para alumnos con discapacidad motora, es decir son mesas con hendidura y reposa pies.

El material didáctico que utilizamos en este aula está dispuesto según ámbitos de trabajo, es decir, para la lectura y la escritura (puzzles de madera con las letras, métodos de lectura, utensilios de escritura con adaptaciones...), en el área de matemáticas (cuerpos geométricos en madera, ábaco, dominós, números de madera con diferentes texturas...), materiales para trabajar la memoria, la psicomotricidad, manipulación... Habrá material que sólo lo utilicen determinados niños y otros que sean compartidos, sobre todo en lo referente a programas de ordenador (ejemplo: Programa Jaws para alumnos con discapacidad visual, el equipo de FM para los alumnos con discapacidad auditiva...).

Todos los alumnos que están en nuestra aula tienen informes de canalización emitido por el Equipo de Orientación Educativa mientras se realiza la Evaluación Psicopedagógica. Dentro de las instrucciones para la cumplimentación del estadillo para el censo del alumnado con Necesidades Educativas Especiales hemos tenido en cuenta del mismo los criterios para determinar en qué categoría han de incluirse los alumnos con NEE que se encuentran en nuestro centro:

- Tres alumnos con anomalías cromosómicas; J.A. está escolarizado en el primer nivel, L. en el segundo nivel del primer ciclo de Educación Primaria y A.M.T. en el primer nivel del segundo ciclo de Educación Primaria.

- P.C. con Trastorno Generalizado del desarrollo está en el primer nivel del tercer ciclo de Educación Primaria.

- R.F. presenta discapacidad mental y está escolarizada en el primer nivel del segundo ciclo de Educación Primaria.

- A.M. tiene discapacidad motora en el segundo nivel del tercer ciclo de Educación Primaria.

- Con trastornos emocionales; tenemos a F.R. que está en el primer nivel del primer ciclo de Educación Primaria y C.G. está en el segundo nivel del tercer ciclo de Educación Primaria.

- E.R. con inteligencia límite que se encuentra escolarizada en el primer nivel del tercer ciclo de Educación Primaria.

- Con discapacidad psíquica por deprivación sociocultural, tenemos a C.L y a J.L. están escolarizados en el segundo nivel del primer ciclo de Educación Primaria.

\section{5.- ESTRUCTURA ORGANIZATIVA.}

El maestro de Apoyo a la Integración.

Desde que la integración ha llegado a nuestro país con la promulgación del Real Decreto 334/1982 de 6 de marzo, la Educación Especial deja de entenderse como la educación de un "tipo de personas" y pasa a considerarse como el conjunto de ayudas necesarias para satisfacer las necesidades especiales de ciertos alumnos/as. Dentro de esas ayudas podemos decir que nos incluimos los maestros de Apoyo a la Integración.

a) Funciones del maestro de Apoyo a la Integración.

¿Qué funciones desempeñamos como maestros de Apoyo a la integración dentro de un centro? 
Según la Orden 9 de Septiembre de 1997 por la que se regulan determinados aspectos sobre la organización y el funcionamiento de los Centros de Educación Infantil y primaria, nuestras funciones se concretan con la coordinación con el tutor de los alumnos con N.E.E. para la realización de las adaptaciones curriculares; la elaboración, seguimiento y realización de programas generales adaptados o de desarrollo individual para la correcta atención del alumnado. En todo momento ha de orientar a los maestros tutores del alumnado atendido así como de colaborar con los mismos en la orientación a los padres y madres; y por último habremos de elaborar el material didáctico que sea necesario para dar una Respuesta Educativa de calidad.

Para que estas funciones se lleven a cabo es necesario que nos marquemos un plan de trabajo que se concretará en el calendario anual.

b) Objetivos generales del Plan de Trabajo

Según nos determinan las funciones que tenemos en el centro y las actuaciones que se concretan en nuestro calendario anual, una vez analizada la realidad física y humana del centro, la diversidad del alumnado que tenemos y la realidad pedagógica que hemos visto en el Proyecto de Centro para esta comunidad Educativa nos exige plantearnos una serie de objetivos en nuestro Plan de Trabajo, estos son:

$\square$ Objetivos de los niveles de concreción curricular:

En la Programación de aula de los tutores de los alumnos con NEE vamos a: asesorar a los tutores en la adaptación del currículo a las necesidades que se presentan; proponer los medios y recursos que sean necesarios; contemplar actividades de las actuaciones educativas para todo el grupo clase; compartir con los tutores la información necesaria para adquirir estrategias metodologías que se adecue a las necesidades educativas de los alumnos; trabajar los temas transversales como concreción de las finalidades educativas y proponer los criterios de evaluación de acuerdo con las necesidades de los alumnos.

$\square$ Objetivos del proceso de enseñanzaaprendizaje. Estos objetivos serán tomados del Proyecto Curricular de Etapa de nuestro centro que son extraídos del Decreto 105/1992 por el que se establece el currículo de Educación Primaria.

\section{6.- DESCRIPCIÓN DEL ALUMNO OBJETO DEL PLAN.}

Lucía es una niña de 8 años, que se encuentra en el segundo nivel del primer ciclo de Educación Primaria. Se encuentra escolarizada en nuestro centro desde los tres años. No promocionó en el último nivel de Educación Infantil.

Tras supervisar la evaluación psicopedagógica de la alumna hemos visto que ésta presenta retraso mental moderado, con hipoacusia ligera de 20 y $30 \mathrm{~dB}$, estrabismo convergente y miopía por lo que se hace necesario la utilización de lentes. Presenta hipotonía muscular que es más acusada en lo miembros inferiores lo que causa unos reflejos débiles y una cara ancha y plana con fisuras palpebrales oblicuas.

Lucía ha recibido atención temprana desde el nacimiento hasta los tres años en el Centro de Valoración y Diagnóstico de la ciudad, desde los tres años acude a una asociación tres veces por semana, en la que recibe clases de refuerzo, logopedia y fisioterapia. Además recibe clases particulares en casa con una logopeda dos veces por semana.

En cuanto a sus aspectos diferenciales en las áreas desarrollo hemos de destacar que el mundo ha de percibirlo y dominarlo por sus sentidos, debemos de proceder a desarrollar las capacidades para ejecutar las tareas constructivas prácticas, potenciar su pensamiento lógicoverbal, para que rebase lo perceptual y de soluciones a pequeños problemas.

En la Atención se debe de procurar una motivación para sobreponerse al estado de apatía que algunas veces presenta la alumna, con lo que le lleva a distraerse con gran facilidad. Nuestra alumna presenta limitaciones importantes en la memoria a corto y a largo plazo sobre todo si la información se presenta de forma auditivoverbal. Suele mejorar con refuerzos visuales complementarios. Lenta recuperación de la información. En la entrada de la información se suelen dar dificultades para la percepción, para la exploración sistemática de la información, en la comprensión de palabras y conceptos, por lo que deberá desarrollarse la adquisición de hábitos a través de la repetición, siendo de gran trascendencia en el logro de la asimilación de conocimientos fundamentales, la repetición de ejercicios lo más conscientes posibles y no de manera mecánica.

Su capacidad de comunicación y el 
desarrollo del lenguaje se ven afectadas, no sólo por sus dificultades para la simbolización y la representación, sino también por aspectos relacionados con la capacidad de emitir y articular palabras: presenta dificultades en la respiración y en la motricidad bucofacial. Tiene numerosas dislalias y un vocabulario muy reducido para su edad. Su nivel comprensivo es más alto que el expresivo. Muestra gran dificultad en el aprendizaje de conceptos que requieran nivel de abstracción.

En el desarrollo motor Lucía presenta una hipotonía muscular con lo que se cansa fácilmente en las tareas que requieran mucho ejercicio físico. Sus movimientos no son del todo ágiles presentando especial dificultad en la motricidad fina.

En el área social nuestra alumna muestra rabietas cuando no se hace lo que ella quiere, no suele tener problemas en la relación con los demás compañeros aunque en algunas ocasiones suele distanciarse del grupo para estar sola. Es muy cariñosa con los demás sobre todo si la elogian con respecto a la ropa que lleva puesta, es muy presumida.

Una vez que hemos descrito como son las diferentes áreas de desarrollo de nuestra alumna nos gustaría establecer una línea base para conocer de qué nivel se parte y así poder planificar las actuaciones educativas en función de ello.

En el Área de Conocimiento del medio, lucía es capaz de distinguir: lo que es un animal de una planta; observa y explora su entorno familiar; conoce las principales dependencias por las que se mueve; reconoce y utiliza los utensilios para la higiene personal y conoce el nombre y el uso de los aparatos electrodomésticos más usados comúnmente (lavadora, lavavajillas, frigorífico,...).

En el Área de lengua castellana y literatura, la alumna conoce el significado de palabras muy usuales; ejecuta mandatos sencillos; responde a preguntas concretas y con poca información; usa palabras frase; le gusta contar cosas que le han pasado y lo hace de forma repetitiva, siempre cuenta lo mismo haciendo uso excesivo de los gestos; reconoce y lee las vocales y las palabras que contengan sílabas directas con $\mathrm{m}$, p y t; coge el lápiz de forma adecuada y la postura, al escribir, es la adecuada; la posición de las manos, al escribir es la correcta y realiza bien los giros; enlaza las letras de cada palabra y el tamaño es irregular al igual que la linealidad al escribir; dibuja cada letra de varios trazos y copia sílabas directas que contengan $\mathrm{m}, \mathrm{p}$ y t, y las palabras que se formen con las mismas.

En el Área de matemáticas, Lucía es capaz de reconocer y reproducir las formas básicas con apoyo visual; cuenta hasta el diez pero hay que seguir trabajando porque lo ha adquirido de forma mecánica y no ha realizado, por tanto un aprendizaje significativo; es capaz de situar actividades de la vida cotidiana en el tiempo: día, noche, mañana y tarde, relacionando el tiempo con distintas actividades relevantes de la vida social (noche-dormir, al levantarse- desayunar, fiesta-no voy al colegio.

\section{Datos familiares.}

Lucía es la menor de tres hermanos (dos niñas y un niño). El padre de la alumna es oficial de correos en el pueblo donde viven y la madre cuida a dos niños pequeños de la vecindad, por lo que Lucía siempre está en contacto con niños pequeños con los que juega por las tardes. Estos se preocupan mucho por su hija e intentan hacer de su hogar un lugar estimulante para la niña aunque a veces tienden a sobreprotegerla. Colaboran en todo momento con el colegio y participan en todas las jornadas y grupos de trabajo que se organizan desde el mismo acerca de las N.E.E. Suelen controlar bastante bien la ansiedad que puede causar la discapacidad de Lucía aunque a veces acuden a los profesionales que intervienen con la misma para recibir consejos. Los dos hermanos mayores se encuentran muy sensibilizados con el problema que presenta Lucía y le intentan ayudar en todo lo que pueden.

\section{7.- PROGRAMCIÓN DE LA RESPUESTA EDUCATIVA.}

Para programar la Respuesta Educativa nos centraremos, como hemos señalado con anterioridad, en Lucía para ello es preciso que nos marquemos una línea de trabajo que comenzará por el análisis de la evaluación psicopedagógica y la realización de la evaluación de en el contexto de enseñanza-aprendizaje descrito en el punto anterior. Fruto de este análisis surge nuestra intervención curricular que en todo momento ha de estar coordinada con el maestro-tutor de nuestros alumnos.

La Respuesta Educativa que nos marquemos para nuestra alumna irá encaminada a conseguir los siguientes aspectos: 


\section{Objetivos.}

Con Lucía nos iniciaremos en el aprendizaje de la lectura y la escritura, la adquisición del vocabulario propio del ciclo, la identificación de las normas, valores y costumbres que le permitan integrarse en el medio en que vive así como establecer las estrategias necesarias que le permitan desarrollar iniciativas conversacionales con sus compañeros con el fin de evitar que la alumna tienda a aislarse. El aprendizaje de los números hasta el 99 también será otro de nuestros objetivos, en cuanto a las operaciones instrumentales básicas enseñaremos a nuestra alumna a sumar y restar sin llevar y a la resolución de problemas sencillos con las mismas operaciones. También trataremos de que la niña reconozca algunas de las figuras geométricas más simples. En todo momento hemos de trabajar la autonomía personal del alumno por medio del conocimiento y desenvolvimiento en el entorno que le rodea. Trataremos de que conozca las dependencias del colegio, de su casa, del barrio en el que vive, las tiendas que forman el mismo, las profesiones más comunes y relacionadas con los miembros de su familia... en definitiva queremos que la alumna desarrolle al máximo las capacidades por medio de la interacción con sus iguales.

Estos objetivos serán marcados desde una perspectiva común entre todos los maestros que trabajan con la niña en el centro escolar, la familia, así como la asociación a la que acude y la logopeda que le da clases particulares.

Todos estos objetivos junto con sus contenidos serán detallados en las unidades didácticas que se presentarán a continuación.

\section{Metodología.}

La metodología que vamos a seguir en nuestra intervención estará conectada en todo momento con una serie de principios generales: partiremos del nivel de desarrollo de Lucía, asegurando aprendizajes constructivos y significativos. En todo momento se proporcionarán estrategias que favorezcan el autoaprendizaje. Utilizaremos la observación, la estimulación sensorial, la manipulación de los objetos, y otras técnicas que son especialmente relevantes para Lucía. Las estrategias que llevemos a cabo para motivar a Lucía se han de centrar en la creación de situaciones que conecten con sus intereses y expectativas, partiendo de sus propias experiencias. Para aprender, Lucía debe de reconocer las dificultades y los errores que comete durante el proceso de aprendizaje cuyo objetivo es poder superarlo y llegar al aprendizaje de la autorregulación. Uno de los instrumentos de los que disponemos para lograrlo es la gestión del aula mediante la estrategia de grupos de trabajo cooperativo, esto permitirá a nuestra alumna estar integrada en un grupo, valorando las diferencias individuales y que los demás alumnos conozcan a nuestra alumna como una más entre ellos.

\section{Actividades.}

Las actividades se adecuarán en todo momento al estilo de aprendizaje y motivación de Lucía. Para nuestra alumna en concreto será necesario plantear actividades que sigan siempre la misma progresión y estructuración para conseguir desarrollar estrategias de auto planificación de sus acciones. Las tareas se analizarán y presentarán paso a paso, siguiendo la técnica de análisis de tareas. Las instrucciones para la realización de las actividades y la explicación de las mismas deben ser cortas claras, concretas y bien pronunciadas. Las actividades y tareas se programarán para responder a objetivos concretos y bien delimitados, concediéndose un poco más de tiempo a nuestra alumna para que pueda responder a las tareas que se proponen, sobre todo si implican operaciones abstractas. Por lo que requerirá más ayuda, más práctica y más tiempo. Partiremos siempre de actividades de manipulación, priorizando la vía visual sobre la auditiva y yendo de lo concreto a lo abstracto, con el fin de que pueda almacenar adecuadamente significado y significante en su memoria a corto plazo. Las actividades de lectura y escritura se plantearán por medio del uso de fotos o pictogramas y cuando sea posible de objetos reales, de manera que la acerquen todo lo que sea posible a su entorno. En cada una de las actividades que programemos irán encaminadas a desarrollar la autonomía personal y social de nuestra alumna por ser esta la finalidad que pretendemos conseguir en nuestra intervención.

\section{Organización del apoyo.}

Este Plan de Trabajo, no se podría considerar como tal si no contemplara la adecuada organización, entendiéndola ésta como una de las medidas que contempla la Atención a la Diversidad. Uno de los requisitos imprescindibles para una adecuada organización del apoyo será determinar el horario en el que Lucía va a estar con el maestro de Apoyo a la Integración que se van a concretar entre la primera y la segunda 
sesión con un total de cinco sesiones semanales en las que se trabajarán lengua y matemáticas

\section{8.- UNIDADES DIDÁCTICAS ADAPTADAS.}

Las unidades didácticas que a continuación se presentan se caracterizan por tener un marcado carácter curricular. En ellas se concretan los objetivos que vamos a trabajar con Lucía, como maestros de Apoyo a la Integración, tanto dentro como fuera del aula ordinaria y los que vamos a desarrollar con el maestro de Audición y Lenguaje, se recogen en las quince unidades didácticas que han sido extraídas de la Programación de Aula del tutor. Estos objetivos han sido elegidos porque creemos que son los más importantes para trabajar con los profesionales que hemos nombrado con anterioridad. Los contenidos se adecuarán a los objetivos. A la hora de formular las actividades es de gran importancia que se tenga en cuenta el ritmo y estilo de aprendizaje de nuestra alumna. Así partiremos de lo concreto para llegar a lo abstracto, de lo manipulativo a lo imaginativo y de la experiencia real a lo imaginario. Al comenzar cada unidad didáctica se realizará una evaluación inicial para ver los conocimientos previos que tiene nuestra alumna en relación con el tema que vamos a trabajar. A lo largo de todas las unidades se llevará a cabo una evaluación continua (esta evaluación se concretará a lo largo de las quince unidades didácticas) con el fin de detectar dificultades y poner medios para perfeccionar aún más la Respuesta Educativa y al terminar el nivel realizaremos una evaluación final para analizar los objetivos que nos hemos marcado con la alumna $y$ los que hemos conseguido para plasmarlo en la Memoria Final de curso, con el objeto de que el año próximo se de continuidad a lo que hemos iniciado este curso con Lucía. Teniendo como objetivo fundamental el desarrollo Integral de la misma.

A continuación presentamos un ejemplo de las unidades didácticas que trabajaríamos con Lucía.

\section{UNIDAD DIDÁCTICA1: “LA VUELTA AL COLE"}

$\square$ Lengua Castellana y Literatura (tabla 1).

\section{$\square$ Matemáticas (tabla 2)}

\section{Criterios de evaluación:}

a. Conocer el vocabulario básico de la unidad, con al menos, cinco palabras de cada familia trabajada.

b. Reconoce los miembros de su familia y comprende, al menos, la relación de parentesco entre los familiares más cercanos.

c. Reconoce, lee y escribe números, por lo menos tres de los cinco propuestos.

\section{UNIDAD DIDÁCTICA 2: "EL OTOÑO YA LLEGÓ”}

$\square$ Lengua Castellana y Literatura (tabla 3).

\section{$\square$ Matemáticas (tabla 4)}

Tabla 1.- Unidad didáctica 1: "la vuelta al cole”.

Lengua Castellana y Literatura.

\begin{tabular}{|c|c|c|}
\hline OBJETIVOS & CONTENIDOS & ACTIVIDADES \\
\hline \begin{tabular}{lrr}
\multicolumn{2}{l}{ 1. Conocer el } & \multicolumn{2}{r}{ vocabulario } \\
básico de & la & unidad \\
utilizando & para & ello \\
pictogramas. & & \\
\end{tabular} & $\begin{array}{l}\text { 1.1. Vocabulario de la unidad } \\
\text { (C). } \\
\text { 1.2. Reconocimiento del } \\
\text { vocabulario básico de la } \\
\text { unidad utilizando pictogramas } \\
\text { (P). }\end{array}$ & $\begin{array}{l}\text { - Relaciona los dibujos con las tarjetas que } \\
\text { contienen las palabras. } \\
\text { - Con las fotografías de la familia } \\
\text { intentaremos que diga los nombres de las } \\
\text { personas que representa, después buscaremos } \\
\text { la tarjeta de su nombre. }\end{array}$ \\
\hline $\begin{array}{l}\text { 2. Expresar oralmente una } \\
\text { acción que le haya sucedido } \\
\text { en el colegio. }\end{array}$ & $\begin{array}{l}\text { 2.1.- Exposición oral de una } \\
\text { acción que le haya sucedido en } \\
\text { el colegio o familia (P). } \\
\text { 2.2.- Conocimiento de la } \\
\text { importancia de expresarse } \\
\text { adecuadamente (A). }\end{array}$ & $\begin{array}{l}\text { - Utilizando el vocabulario que hemos } \\
\text { aprendido intentaremos que el niño exprese } \\
\text { lo que le gusta y lo que no le gusta del } \\
\text { colegio. } \\
\text { - Le mostraremos al niño palabras } \\
\text { relacionadas con el colegio que tendrá que } \\
\text { reconocer y nombrar. }\end{array}$ \\
\hline $\begin{array}{l}\text { 3. Comprender una frase en } \\
\text { la que intervenga un } \\
\text { personaje. }\end{array}$ & $\begin{array}{l}\text { 3.1. El texto narrativo }(\mathrm{C}) \text {. } \\
\text { 3.2. Reconocimiento del } \\
\text { personaje que hay en una frase } \\
\text { (P). }\end{array}$ & $\begin{array}{l}\text { - Le ponemos varios pictogramas de } \\
\text { personajes y entre ellos ha de buscar el que } \\
\text { está en la frase. } \\
\text { - Con la frase de referencia el niño ordenará } \\
\text { los pictogramas que le demos del mismo. }\end{array}$ \\
\hline
\end{tabular}


Tabla 2.- Unidad didáctica 1: "la vuelta al cole”.

Matemáticas.

\begin{tabular}{|c|c|c|}
\hline OBJETIVOS & CONTENIDOS & ACTIVIDADES \\
\hline $\begin{array}{l}\text { 1. Reconocer, leer y escribir } \\
\text { números hasta el } 10 .\end{array}$ & $\begin{array}{l}\text { 1.1. Los números hasta el } 10 \\
\text { (C). } \\
\text { 1.2. Reconocimiento, lectura, } \\
\text { escritura y descomposición de } \\
\text { números hasta el } 10 \text {. } \\
\text { 1.3. Valorar la utilidad del } \\
\text { dominio de los números (A). }\end{array}$ & $\begin{array}{l}\text { - Realizaremos conjuntos de objetos y junto a } \\
\text { ellos en otra columna el número para que el } \\
\text { niño los vaya uniendo. } \\
\text { - Realizamos grupos de objetos con números } \\
\text { hasta el } 10 \text {. } \\
\text { - Con una serie de números dados hasta el } 10 \\
\text { vamos a escribirlo (por medio de la unión de } \\
\text { puntos o copiándolos) con letra y luego con } \\
\text { número. }\end{array}$ \\
\hline $\begin{array}{l}\text { 2. Comprender el concepto } \\
\text { de unidad. }\end{array}$ & $\begin{array}{l}\text { 2.1. La unidad (C). } \\
\text { 2.2. Reconocimiento de la } \\
\text { unidad (P). }\end{array}$ & $\begin{array}{l}\text { - Recortamos cuadraditos de cartón del } \\
\text { mismo tamaño y los forramos de cartulina de } \\
\text { un mismo color, estas son las unidades. } \\
\text { - Con cajas de zapatos vamos a poner en el } \\
\text { horizontal los números del } 1 \text { al } 10 \text { y dentro } \\
\text { vamos a introducir las unidades que } \\
\text { correspondan. }\end{array}$ \\
\hline $\begin{array}{l}\text { 3. Resolver sumas sin llevar } \\
\text { por medio de la } \\
\text { manipulación de objetos. }\end{array}$ & $\begin{array}{l}\text { 3.1. La suma }(\mathrm{C}) \text {. } \\
\text { 3.2. Realización de sumas con } \\
\text { números del } 1 \text { al } 10(\mathrm{P}) .\end{array}$ & $\begin{array}{l}\text { - Por medio de objetos de la clase vamos a } \\
\text { realizar sumas con los mismos. } \\
\text { - Iremos al mercadillo del pueblo y } \\
\text { realizaremos una serie de compras que } \\
\text { implique la suma. }\end{array}$ \\
\hline
\end{tabular}

Tabla 3.- Unidad didáctica 2: “el otoño ya llegó.

Lengua Castellana y Literatura.

\begin{tabular}{|c|c|c|}
\hline OBJETIVOS & CONTENIDOS & ACTIVIDADES \\
\hline $\begin{array}{l}\text { 1.- Conocer el vocabulario } \\
\text { básico de la unidad } \\
\text { utilizando para ello } \\
\text { pictogramas (el otoño). }\end{array}$ & $\begin{array}{l}\text { 1.1.- Vocabulario de la unidad: } \\
\text { el otoño (C). } \\
\begin{array}{ll}\text { 1.2.- Identificación el } \\
\text { vocabulario básico de la } \\
\text { unidad por medio de } \\
\text { pictogramas (P). }\end{array}\end{array}$ & $\begin{array}{l}\text { - Relaciona los dibujos con las palabras que } \\
\text { tengan que ver con el otoño y la montaña. } \\
\text { - Salimos al patio del colegio y observamos } \\
\text { el paisaje del otoño para trabajar el } \\
\text { vocabulario reconociendo las palabras que } \\
\text { hemos aprendido., después realizamos un } \\
\text { mural sobre lo que hemos visto. }\end{array}$ \\
\hline $\begin{array}{l}\text { 2. Reconocer el personaje } \\
\text { con la acción que } \\
\text { desarrollen en la frase. }\end{array}$ & $\begin{array}{l}\text { 2.1. La frase }(\mathrm{C}) \text {. } \\
\text { 2.2. Identificación del } \\
\text { personaje con la acción que } \\
\text { desarrolla en la frase }(\mathrm{P}) \text {. }\end{array}$ & $\begin{array}{l}\text { - Buscamos la acción que realiza el personaje } \\
\text { en el texto por medio de las fotografías. } \\
\text { - Relaciona el personaje (fotografía) con la } \\
\text { acción que desarrolla (que estará escrita. }\end{array}$ \\
\hline $\begin{array}{l}\text { 3. Conocer los signos de } \\
\text { interrogación a través de las } \\
\text { situaciones en que se } \\
\text { utilizan. }\end{array}$ & $\begin{array}{l}\text { 4.1. Los signos de } \\
\text { interrogación (C). } \\
\text { 4.2. Reconocimiento de los } \\
\text { signos de interrogación en } \\
\text { cada una de las situaciones que } \\
\text { se vayan a utilizar (P). }\end{array}$ & $\begin{array}{l}\text { - Conoceremos los signos de interrogación y } \\
\text { algunas de las situaciones en las que se } \\
\text { utilizan como por ejemplo: para preguntar la } \\
\text { hora... } \\
\text { - Identificación del signo con la entonación } \\
\text { del mismo. } \\
\text { - Escribimos el signo delante y detrás de la } \\
\text { frase o palabra. }\end{array}$ \\
\hline $\begin{array}{l}\text { 4. Elaborar listas de } \\
\text { palabras (otoño). }\end{array}$ & $\begin{array}{l}\text { 5.1. Las listas de palabras(C). } \\
\text { 5.2. Elaboración de listas de } \\
\text { palabras (otoño) }(\mathrm{P}) \text {. }\end{array}$ & $\begin{array}{l}\text { - Haremos un mural con las fotos del otoño y } \\
\text { debajo pondremos la palabra que } \\
\text { corresponda. } \\
\text { - Con hojas secas realizaremos un póster } \\
\text { dibujando un árbol y alrededor los frutos que } \\
\text { se recogen en otoño. }\end{array}$ \\
\hline
\end{tabular}


Tabla 4.- Unidad didáctica 2: “el otoño ya llegó.

Matemáticas.

\begin{tabular}{|c|c|c|}
\hline OBJE & CONTENIDOS & ACTIVIDADES \\
\hline $\begin{array}{l}\text { 1. Reconocer, leer y } \\
\text { escribir números hasta } \\
\text { el } 20 \text {. }\end{array}$ & $\begin{array}{l}\text { 1.1. Los números hasta el } 20(\mathrm{C}) \text {. } \\
\text { 1.2. Reconocer, leer y escribir } \\
\text { números hasta el } 20(\mathrm{P}) \text {. }\end{array}$ & $\begin{array}{l}\text { - Realizaremos conjuntos de objetos y junto } \\
\text { a ellos en otra columna el número para que el } \\
\text { niño los vaya uniendo. } \\
\text { - Con una serie de números dados hasta el } 10 \\
\text { vamos a escribirlo (por medio de la unión de } \\
\text { puntos o copiándolos) con letra y luego con } \\
\text { número. }\end{array}$ \\
\hline $\begin{array}{l}\text { 2. Comprender los } \\
\text { conceptos de unidades } \\
\text { y decenas. }\end{array}$ & $\begin{array}{l}\text { 2.1. Las decenas y las unidades (C). } \\
\text { 2.2. Reconocimiento de las unidades } \\
\text { y las decenas (P). }\end{array}$ & $\begin{array}{l}\text { S a averiguar cuantas decenas tiene el } \\
10 \text { y el } 20 \text {. Cuando lleguemos al } 10 \\
\text { lades las cambiamos por las decenas y } \\
\text { mos descomponiendo todos los } \\
\text { s. }\end{array}$ \\
\hline $\begin{array}{l}\text { 3. Sumar } \\
\text { un dígito. }\end{array}$ & $\begin{array}{l}\text { 3.1. La suma }(\mathrm{C}) \\
\text { 3.2. Cálculo de sumas de números } \\
\text { de un dígito }(\mathrm{P}) \text {. }\end{array}$ & $\begin{array}{l}\text { - Escribimos en el cuadernos las sumas que } \\
\text { vayamos haciendo paso a paso con los } \\
\text { objetos reales, identificando el signo + con la } \\
\text { adición de objetos. } \\
\text { - Representamos las sumas realizadas con } \\
\text { dibujos de conjuntos, dibujando el objeto y } \\
\text { colocando debajo el número. }\end{array}$ \\
\hline \multicolumn{3}{|c|}{ 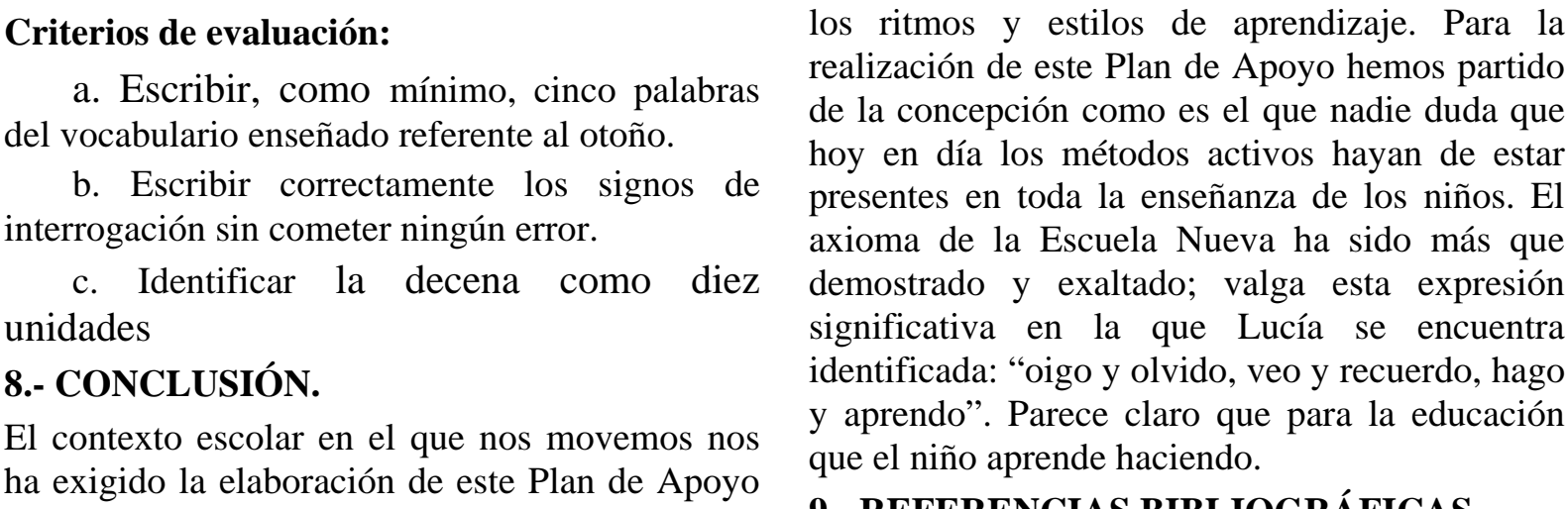 } \\
\hline
\end{tabular}
como una de las medidas más importantes para dar Respuesta a las Necesidades de nuestros alumnos, que en definitiva son los grandes beneficiarios de su esfuerzo y de nuestro trabajo. En este artículo hemos concretado las actuaciones a llevar a cabo por todos lo profesionales que intervienen directa o indirectamente en el proceso de enseñanzaaprendizaje con nuestros alumnos con Necesidades Educativas Especiales, estableciendo para ello una coordinación entre los grandes contextos que hacen que el desarrollo del alumno sea su gran meta. Una meta que se consigue alcanzar si se aúnan los esfuerzos de todos: familia, profesores y en definitiva la sociedad en general. Para la intervención concreta con Lucía hemos tomado como base el currículo ordinario haciendo las correspondientes adaptaciones con el fin de que se ajuste a las características de la misma. Con lo que hemos desarrollado una propuesta eminentemente curricular conectada con la programación de aula de su grupo clase, respetando en todo momento

\section{9.- REFERENCIAS BIBLIOGRÁFICAS.}

Ausubel, D. P. (1975). Psicología educativa. México: Trillas.

Doyle, W. (1977). Paradigms of research on teacher effectiveness. En Shulman, L. (ed): Review of Research in Education, Itasca, Ill: Peacock.

Froebel, F. (1826). La educación del hombre. Madrid: D. Jorro

Norman, D. A. (1987). Perspectivas de las ciencias cognitivas. Barcelona: Paidós.

Pestalozzi, J. E. (1801). Cómo Gertrudis enseña a sus hijos. México: editorial Porrúa.

Piaget, J. (1981). Seis estudios de psicología. Barcelona: Seis Barral.

Vigotsky, L. S. (1985). Pensamiento y lenguaje. Buenos Aires: La Pléyade.

Bibliografía básica para consulta.

Garrido Landivar, J. \& Santana Hernandez, R. (1999). Cómo elaborar adaptaciones curriculares de centro, de aula $e$ 
individuales. Madrid: CEPE.

Verdugo, M. A. (1990). Alumnos con discapacidad. Madrid: Alianza Editorial.

Marchesi, A., Coll, C. \& Palacios, J. (1999). Desarrollo psicológico y educación. Vol. III. Madrid: Alianza Editorial.

Lorenzo, M. \& Sola, T. (2002). Didáctica y organización de la Educación Especial. Madrid: Dykinson.

Mayor, J. (1998). Manual de Educación Especial. Madrid: Anaya.

\section{Referencias legislativas básicas}

- Currículos de las Etapas de E.I. y E.P. tenemos respectivamente los D.107 y 105 de 1992 de 9 de Junio.

- Procedimiento de Diseño, Desarrollo y Aplicación de Adaptaciones Curriculares en los centros docentes de EI, EP y E.S.O.: Orden 13 de Julio de 1994

- Orden de 18 de Noviembre de 1996 sobre Evaluación de las enseñanzas de Régimen General, que establece los criterios de evaluación para el acnee a partir de la adecuación de los criterios generales.

- R.D. 696/1995 de 28 de Abril que deroga el 334/1985 en el que se disponen los aspectos referidos a la ordenación de la atención educativa a los acnee, del que se deriva la Orden de 14 de Febrero de 1996 sobre la realización de la Evaluación Psicopedagógica y el Dictamen de Escolarización, que establece los criterios de Escolarización para estos alumnos.

- Ley orgánica 2/2006, de 3 de mayo, de educación: los principios que presiden esta ley son: exigencia de proporcionar una educación de calidad a todos los ciudadanos de ambos sexos, en todos los niveles del sistema educativo; necesidad de que todos los componentes de la comunidad educativa colaboren para conseguir ese objetivo tan ambicioso; compromiso decidido con los objetivos educativos planteados por la Unión Europea para los próximos años.

En Andalucía:

- Ley 1/1999 de 31 de Marzo de Atención a las personas con Discapacidad, que establece la atención integral de este colectivo en los ámbitos sociales, laboral, educativo, etc.

- Ley 9/1999 de 18 de Noviembre, de SOLIDARIDAD EN LA EDUCACIÓN, que trata de mejorar las condiciones de escolarización de los alumnos con necesidades educativas especiales.

- Para desarrollar esta última ley surge el D.147/2002 de 14 de Mayo por el que se establece la ordenación de la atención educativa de los alumnos con necesidades educativas especiales asociadas a sus capacidades personales, dejando así de tener validez en Andalucía el R.D. 696/95.

- Este Decreto 147 da lugar a la Orden de 19 de Septiembre de 2002 que regula la realización de la evaluación psicopedagógica y el Dictamen de escolarización perdiendo urgencia la orden del 14 de febrero anteriormente citada.

Desarrollo posterior a la LO.E. (ámbito estatal):

- LEY 39/2006, de 14 de diciembre, de Promoción de la Autonomía Personal y Atención a las personas en situación de dependencia.

- R.D. 1630/2006, de 29 de diciembre, por el que se establecen las enseñanzas mínimas del segundo ciclo de Educación infantil.

- $\quad$ R. D. 1513/2006, de 7 de diciembre, por el que se establecen las enseñanzas mínimas de la Educación Primaria.

- $\quad$ R. D. 1631/2006, de 29 de diciembre, por el que se establecen las enseñanzas mínimas correspondientes a la Educación Secundaria Obligatoria.

Desarrollo posterior a la L.O.E. en la comunidad autónoma andaluza:

- Ley 17/2007, de 10 de diciembre, de Educación de Andalucía: la Junta de Andalucía envió a los centros educativos en el curso 2005/2006 una propuesta para el debate de una ley de educación para Andalucía. La Ley de Educación de Andalucía (LEA), primera norma de ámbito autonómico que regulará el sistema educativo en una comunidad autónoma, fue aprobada por el Parlamento andaluz el pasado 21 de noviembre de 2007. 